\title{
A relevância do grupo de gestantes na Atenção Primária à Saúde: uma revisão da literatura
}

The relevance of the group of Pregnant Women in Primary Health Care: a literature review

La relevancia del grupo de gestantes en la Atención Primaria de Salud: una revisión de la literatura

Ezilaine Albino Monteiro Santos ${ }^{1 *}$, Lorrany Vieira de Lima ${ }^{1}$, Jacqueline Rodrigues do Carmo Cavalcante ${ }^{2}$, Mônica Santos Amaral ${ }^{3}$.

\section{RESUMO}

Objetivo: Analisar a produção bibliográfica sobre a importância dos grupos de gestantes no âmbito da Atenção Primária à Saúde. Revisão bibliográfica: A prática da educação em saúde no âmbito da Atenção Primária é uma estratégia essencial, pois permite que os profissionais de saúde e a população se aproximem, contribuindo para uma assistência integral, de qualidade e humanizada. Atividades de grupo com gestantes proporcionam um ambiente dinâmico e objetiva a promoção da saúde integral, individual-coletiva das gestantes qualificando o pré-natal. As gestantes percebem as reuniões como um apoio para diminuir as suas angústias, ansiedade, espaço para trocar experiências, articular o saber popular com o saber científico e expor seus questionamentos. Observou-se que a literatura aborda a importância do trabalho dos profissionais voltado ao pré-natal, enf atizando a necessidade de práticas no campo da educação em saúde que favoreçam a atenção integral às gestantes. Considerações finais: Ficou evidente que a existência e a implantação de grupos de gestantes nos serviços de saúde são de fundamental relevância para dar apoio e preparar a gestante e seus familiares para o cuidado no período gravídico, puerperal e no cuidado com a criança, o que fortalece vínculos familiares e promove a saúde do binômio mãe-filho.

Palavras-chave: Gravidez, Educação em saúde, Pré-natal, Enfermagem.

\begin{abstract}
Objective: To analyze the literature on the importance of groups of pregnant women in Primary Health Care. Bibliographic review: The practice of health education within the scope of Primary Care is an essential strategy, as it allows health professionals and the population to get closer, contributing to comprehensive, quality and humanized care. The group of pregnant women is a dynamic environment, which aims to promote integral, individual-collective health of pregnant women, qualifying prenatal care. It was found that the pregnant women saw the meetings as a support to reduce anguish, anxiety, exchange experiences, articulate popular knowledge with scientific knowledge and expose their questions. It was observed that the literature addresses the importance of professionals' work in prenatal care, emphasizing the need for practices in the field of health education that favor comprehensive care for pregnant women. Final considerations: It was evident that the existence and implementation of groups of pregnant women in the health services is of fundamental importance to support and prepare the pregnant woman and her family forcare in the pregnancy and puerperal period and in the care of the child, which strengthens family bonds and promotes the health of the motherchild binomial.
\end{abstract}

Key words: Pregnancy, Health education, Prenatal, Nursing.

\footnotetext{
${ }_{1}$ Secretaria Municipal de Saúde, Jataí - GO. *E-mail: ezilaine_monteiro@hotmail.com

2 Universidade Federal de Jataí, Jataí- GO.

${ }^{3}$ Centro Universitário de Mineiros (Unifimes), Mineiros - GO.
} 


\section{RESUMEN}

Objetivo: Analizar la literatura sobre la importancia de los grupos de gestantes en la Atención Primaria de Salud. Revisión bibliográfica: La práctica de la educación en salud en el ámbito de la Atención Primaria es una estrategia fundamental, ya que permite el acercamiento de los profesionales de la salud y la población, contribuyendo a una atención integral, de calidad y humanizada. El grupo de gestantes es un ambiente dinámico, que tiene como objetivo promover la salud integral, individual-colectiva de las gestantes, calificando la atención prenatal. Se constató que las gestantes vieron los encuentros como un apoyo para disminuir la angustia, la ansiedad, intercambiar experiencias, articular saberes populares con saberes científicos y exponer sus interrogantes. Se observó que la literatura aborda la importancia del trabajo de los profesionales en el prenatal, destacando la necesidad de prácticas en el campo de la educación en salud que favorezcan la atención integral a la gestante. Consideraciones finales: Se evidenció que la existencia e implementación de grupos de gestantes en los servicios de salud es de fundamental importancia para apoyar y preparar a la gestante y su familia para la atención en el período gestacional y puerperal y en el cuidado del niño, lo que fortalece los lazos familiares y promueve la salud del binomio madre-hijo.

Palabras clave: Embarazo, Educación en salud, Prenatal, Enfermería.

\section{INTRODUÇÃO}

Práticas de educação em saúde que desde a antiguidade eram majoritariamente voltadas para o fortalecimento do autoritarismo biomédico, hegemônico, hospitalocêntrico e curativista, que na maioria das vezes buscavam apenas o cumprimento de medidas sanitárias opressivas, por vezes impostas à população, ou até mesmo nem realizadas, estão sendo desencorajadas. Como resultado de lutas de diversos atores sociais e pela busca desses atores pela real (re)democratização da saúde, práticas tradicionalistas vêm sendo substituídas por modelos promotores da saúde que buscam substituir as práticas tradicionais de educação em saúde em ações que favoreçam o acolhimento integral dos usuários do Sistema Único de Saúde (SUS) de acordo com as próprias demandas de saúde da coletividade (VASCONCELOS EM, 2017).

Graças a esforços coletivos e com a implantação do Programa de Saúde da Família denominado hoje Estratégia Saúde da Família (ESF) dentro da Atenção Primária à Saúde (APS), a equipe multiprofissional em saúde tem a oportunidade de romper com laços do passado e proporcionar o acompanhamento das gestantes acolhidas diariamente no SUS. Na ESF, o enfermeiro, enquanto membro da equipe desenvolve atividades educativas, atua como facilitador do processo de aprendizagem na medida em que percebe os conflitos e interesses que influenciam os modos de vida da população a qual atende (ALVES GG e AERTS D, 2011).

A APS constitui a principal porta de entrada do SUS, tornando-se uma ferramenta fundamental no âmbito do Sistema, com resultados positivos para a melhoria da saúde, o que favorece o acesso da população aos serviços of ertados pelo SUS (SANTOS EAM, et al., 2019; COUTINHO LRP, et al., 2015).

A prática da educação em saúde no âmbito da APS é um mecanismo essencial, pois permite que os profissionais de saúde e a população se aproximem, contribuindo para uma assistência integral, de qualidade e humanizada. Neste sentido, a educação em saúde é uma das importantes atribuições do enfermeiro dent ro da ESF, objetivando a promoção, prevenção, manutenção e recuperação da saúde (REBERTE LM e HOGA LAK, 2005).

Neste contexto, o enfermeiro possui como instrumento de educação em saúde, o trabalho grupal, isto é, a formação de grupos que permitem a troca de experiências, formação de vínculos entre os participantes, além da transmissão e disseminação de conhecimentos, fortalecendo as potencialidades de cada participante (CAMILO BS, et al., 2014).

O grupo de gestantes é um espaço micro e dinâmico, que objetiva a promoção da saúde de forma integral, individual e coletiva mediada pelas interações que nele ocorrem, qualificando positivamente o pré-natal. As conversas, momentos de interação entre as gestantes e os diversos profissionais da rede de assistência à saúde favorecem o diálogo que possibilita o desenvolvimento de ações promotoras da saúde, com 
repercussões desse processo nas dimensões individuais e coletivas (CAMILO BS, et al., 2014; DELFINO MRR, et al., 2004).

Desse modo, durante o período gestacional, a mulher passa por profundas e intensas transformações físicas e emocionais, estando as mesmas intimamente relacionadas. Assim, tantas modificações fazem com que a gestante, juntamente com seu parceiro e familiares, vivam uma fase de adaptação do seu novo papel diante da sociedade, gerando ansiedade e medo para quem a vive (MALUMBRES PC e BARRETO ICHC, 2016; SARTORI GS e VAN DER SAND ICP, 2004).

O desenvolvimento de grupo de gestantes é um recurso importante para promover uma assistência prénatal de qualidade, uma vez que tais grupos promovem um atendimento individualizado e integral das necessidades da gestante, seu parceiro e familiares. Em geral, esses grupos são desenvolvidos com o objetivo de diminuir ansiedades e medos relativos ao período gestacional, complementar o atendimento realizado nas consultas, além de melhorar a adesão desse público aos hábitos considerados mais saudáveis (HOGA LAK e REBERTE LM, 2007).

Diante do exposto, objetivou-se com este estudo analisar a produção bibliográfica sobre a importância dos grupos de gestantes no âmbito da Atenção Primária em Saúde por meio da revisão bibliográfica e descrição narrativa dos achados.

\section{REVISÃO BIBLIOGRÁFICA}

\section{Educação em saúde para grupos de gestantes no âmbito da Atenção Primária à Saúde}

Com o objetivo de realizar o manejo adequado das gestantes na Rede de Atenção em Saúde (RAS), nela a Atenção Primária à Saúde, caracterizada como porta de entrada preferencial da gestante e ordenadora do cuidado, deve-se acolher e garantir o manejo adequado desse público dentro do sistema de saúde de acordo com as suas próprias demandas de saúde (BRASIL, 2019).

Para que esse objetivo seja alcançado, o Ministério da Saúde (MS) tem aconselhado que os profissionais que trabalham no SUS tenham em mente os determinantes e condicionantes de saúde presentes na vida das gestantes, assim como, os fatores que podem gerar riscos para a gestação, como o tabagismo, inatividade física, uso de drogas lícitas ou ilícitas, violência doméstica, condições de trabalho desfavoráveis, insuficiência sócio familiar, dificuldade em navegar pelos sistemas de saúde, entre outros; e que os mesmos trabalhem com vistas a minimizar as iniquidades e vulnerabilidades que afetam o estado de saúde desse público (BRASIL, 2019).

Assim, de forma contínua e articulada, medidas de promoção e prevenção de agravos à saúde a esse público devem ser identificadas e monitoradas, sendo a educação em saúde em grupo, uma forte aliada para a construção de vínculo e o acompanhamento da gestante conforme as suas necessidades. A educação em saúde então é um caminho possível é fortemente recomendado pelo MS (BRASIL, 2019).

O MS ressalta que devem ser garantidos pelo Sistema os serviços essenciais para a promoção do cuidado às gestantes. Dentre eles, temos o acompanhamento pré-natal e puerpério com equipes de profissionais competentes que acompanham e elaboram planos de cuidados e autocuidado apoiado em ações de educação em saúde que focam nas necessidades específicas e na melhoria de vida e do cuidado prestado às gestantes (BRASIL, 2019).

Nesse contexto, a educação em saúde se caracteriza como um conjunto de ações que objetivam aumentar a autonomia das gestantes referente ao seu autocuidado, com temas voltados para a saúde individual como também coletiva, de acordo com as necessidades e demandas trazidas aos profissionais/serviços de saúde, a fim de proporcionar atenção em saúde qualificada (BRASIL, 2013).

A educação em saúde é uma prática promotora da saúde que favorece o diálogo entre os profissionais da saúde e as gestantes que se beneficiam dos serviços ofertados pelo sistema público de saúde brasileiro, 0 SUS. Esses diálogos proporcionam a troca, compartilhamento e valorização dos saberes, se materializando como espaços coletivos de cuidado que podem e devem ser norteados pelo compromisso em propiciar a 
saúde como direito, sendo essas ações orientadas por princípios da educação popular em saúde, como os de amorosidade e emancipação, na busca compartilhada por conhecimento e soluções necessárias de acordo com as necessidades do grupo (BRASIL, 2014).

Assim, a educação em saúde voltada aos usuários do SUS, sobretudo as gestantes podem e devem acontecer em diferentes espaços, como em salas de espera dos serviços de saúde, UBS, igrejas, praças, escolas, espaços co munitários etc.; e podem envolver diferentes procedimentos metodológicos, como rodas de conversas, palestras, debates, diálogo aberto, consultas individuais (CAVALCANTE JRC, 2019).

De acordo com os princípios e diretrizes do SUS as práticas/ações educativ as podem ser realizadas e (re)orientadas por ações/práticas de educação em saúde, educação permanente em saúde e educação popular em saúde, sendo elas caracterizadas pelas concepções de educação em saúde, educação popular em saúde e educação permanente em saúde (BRASIL 2013; BRASIL, 2009; BRASIL, 2007). A educação em saúde se caracteriza como o conjunto de práticas educativas em saúde que buscam a autonomia dos usuários do sistema público de saúde de modo transversal com articulação entre todos os níveis de atenção do SUS (BRASIL, 2013).

No que concerne à Educação Permanente em Saúde as práticas educativas são planejadas e embasadas na problematização da realidade dos serviços de saúde, assim como, dos usuários do SUS; há a integração entre o ensino, serviço, gestão e comunidade transformando os espaços organizacionais e a atuação dos respectivos profissionais a favor da coletividade (BRASIL, 2009). A Educação Popular em Saúde propaga um novo olhar e uma nova roupagem no desenvolvimento de ações voltadas para a educação em saúde no SUS, pois valoriza a cultura popular, com as suas diversas expressões de arte, cuidado, controle social com vistas à autonomia e gestão participativa (BRASIL, 2007).

Especificamente para as gestantes, o MS recomenda que as ações educacionais devam fortalecer o autocuidado e apoiá-las ao gerenciamento de suas condições, conhecimento e avaliação de sua saúde, planejamento de vida e fortalecimento das suas relações familiares e sociais visando o apoio a esse público. Diversos temas podem ser abordados, entre eles alimentação, dinâmica familiar, atividade física, autoestima e autoimagem, atividade sexual, cuidados de higiene pessoal e doméstica, dentre outros. Nesse contexto, o profissional enfermeiro que integra a equipe da APS exerce competências específicas do cargo em que ocupa tendo em vista fortalecer e promover a educação em saúde no âmbito do SUS de forma assertiva e como já tem preconizado o SUS (BRASIL, 2019).

\section{Importância das reuniões e atividades grupais para as gestantes no âmbito da APS}

Reuniões e o desenvolvimento de atividades grupais com as gestantes no âmbito da APS são de suma importância da realização de encontros grupais com gestantes, visto que esses espaços promovem a discussão de assuntos referentes à gravidez, que podem ser pouco abordados durante consultas de pré-natal (BRASIL, 2019). As gestantes percebem as reuniões como um método de apoio para diminuir as suas angústias e ansiedade, trocar experiências, associar e discernir o saber popular com o saber científico e expor seus questionamentos (HERBAS DTA, 2014).

Nesse sentido, a educação em saúde com grupos de gestantes favorece o empoderamento dessas mulheres. Pois aumenta sua percepção sobre o tema, com metodologias ativas que promovem a troca de saberes e práticas entre os profissionais e os participantes dos grupos educativos, fortalecendo 0 conhecimento e promovendo habilidades e o autocuidado, na qual, cada participante acrescenta um pouco da sua vivência e experiência na temática abordada (CAMILO BS, et al., 2014; NUNES GP, et al., 2017).

Ademais, os grupos de gestantes favorecem para a desmistificação de crenças e mitos relativos ao período gestacional, relacionado aos cuidados ao parto e puerpério além dos cuidados com o bebê, e assim, compreender de forma mais clara, as mudanças ocorridas em todas essas etapas supracitadas (NUNES GP, et al., 2017).

Compartilhar emoções, mediante grupos de gestante é benéfico para que outras mulheres dividam seus medos, diferenças, receios, angústias e se sintam mais serenas ao perceberem que outras gestantes 
partilham dos mesmos sentimentos, o que propicia maior troca de conhecimento, aumentando a confiança e segurança entre as participantes (HERBAS DTA, 2014).

Nesse contexto, a participação dos parceiros nas consultas de pré-natal é fundamental para o casal. Sinalizando que a gestação faz parte da vida tanto da mulher, quanto do parceiro. Dessa forma, no período gravídico ocorrem mudanças fisiológicas e psicológicas motivadas por um processo de adaptação, compartilhado entre os cônjuges. Dessa maneira, a participação do parceiro nas consultas de pré-natal deve ser motivada por todos os profissionais de saúde que prestam assistência na Atenção Primária à Saú de, pois possibilita às gestantes maior segurança, além de aumentar o conhecimento do casal com os cuidados materno e infantil (CAMILO BS, et al., 2014).

Permanece a necessidade de apoio do companheiro e demais familiares para a gestante, uma vez que a gestação é uma etapa em que ocorrem várias modificações estruturais e psicológicas e que necessita de amplo apoio emocional (DOMINGUES F, et al., 2018).

\section{Relevância da educação em saúde para os profissionais que atuam nos grupos de gestantes}

Observa-se que o trabalho dos profissionais de saúde na atenção com o pré-natal, deverá enfatizar práticas de educação em saúde que favoreçam a assistência integral e de excelência. O desenvolvimento de grupo de gestantes facilita a aceitação de novas práticas baseadas na promoção da saúde, como foco não apenas na prevenção e tratamento de intercorrências gestacionais, mas também no fortalecimento do vínculo entre as gestantes e os profissionais de saúde (BRASIL, 2019).

Nessa conjuntura, os profissionais de saúde dentro dos grupos educativos têm a função de repassar e trocar seus conhecimentos facilitando o aprendizado, o que promove a confiança e troca de experiências e saberes populares e científicos favorecendo o cuidado integral (VIEIRA AN, et al., 2019; MALUMBRES PC e BARRETO ICHC, 2016).

O grupo de gestantes na Atenção Primária é um lugar grandioso para as trocas de saberes, experiências e fortalecimento de vínculo entre a população e os profissionais de saúde, o que favorece a promoção da saúde, a qualidade do pré-natal e a construção de conhecimentos coletivos (NUNES GP, et al., 2017). As reuniões em grupos com as gestantes possibilitam aos profissionais de saúde a criação de um espaço para conhecer e compreender de perto as alterações que acontecem durante a gestação, bem como, a realização do atendimento assistencial a essa população, o que possibilita também, a busca por conhecimentos e levantamento das dúvidas mencionadas pelas gestantes (BRASIL, 2019).

O desenvolvimento do grupo de gestantes é uma ferramenta fundamental para promover o atendimento integral e humanizado da mulher, seu parceiro e demais familiares no período gravídico e puerperal. Além disso, os grupos possibilitam a troca de conhecimento/vivência de pessoas com histórias de vida diferentes, porém com as mesmas dúvidas e com interesses análogos que no coletivo há somatório de saberes e para, além disso, desvendar suas angústias e restrições, favorecendo a tomada de decisões (DOMINGUES F, et al., 2018).

A dinâmica em grupo é utilizada como ferramenta pelo profissional de saúde para melhora na adesão à assistência ao pré-natal, o que estimula as gestantes a exporem suas dúvidas e inquietações, as quais muitas vezes não são sanadas durante as consultas de pré-natal, mas são esclarecidas durante o desenvolver do grupo devido a essa troca de informações (CAMILO BS, et al., 2014; MALUMBRES PC e BARRETO ICHC, 2016).

Ações educativas com grupo de gestantes possibilitam a articulação entre o conhecimento teórico e o conhecimento prático, sendo mediadora do atendimento integral à saúde da mulher. Permanece a importância da articulação entre ensino e serviço, pois a vivência conjunta de projetos permite que os estudantes se integrem com os profissionais que estão atuando e assim podem perceber o papel real destes profissionais no contexto da Atenção Básica (BRASIL, 2007).

Nessa perspectiva, salienta-se a importância da junção do conhecimento prático com o conhecimento teórico para processos educativos sistematizados, haja vista as necessidades de saúde da comunid ade. 
Assim, somando e aperfeiçoando o processo de ensino-aprendizagem, promovendo maior qualidade da assistência ao pré-natal (VIEIRA NA, et al., 2019).

\section{CONSIDERAÇÕES FINAIS}

A existência de grupos de gestantes é de fundamental importância para dar apoio e preparar a gestante e seus familiares para o cuidado no período gravídico, puerperal e no cuidado com a criança, fortalecendo vínculos familiares e a promoção da saúde do binômio mãe-filho. Observa que o enfermeiro como integrante da equipe e parte desse processo devem buscar cada vez mais estratégias inovadoras que levem essas mulheres aos serviços de saúde o mais precocemente possível, o que dará visibilidade à importância do acompanhamento e da adesão ao pré-natal o mais rápido possível, e assim, promover o acolhimento à gestante e a sua família. A abordagem das vantagens e das dificuldades que possam ocorrer durante a gestação, considerando os conhecimentos prévios, as expectativas e os sentimentos que as levam a se sentirem mais seguras devem ser estimuladas, rumo a superação das possíveis adversidades do período gestacional, parto, amamentação e cuidados com o recém-nascido que poderão surgir. Sendo assim, faz-se cada vez mais necessário, a realização de atividades grupais com as gestantes no âmbito da Atenção Primária à Saúde, na qual, os profissionais de saúde, responsáveis pelo acompanhamento do pré-natal, atuem sanando dúvidas, oferecendo uma assistência integral e atuando em ações de promoção e prevenção da saúde.

\section{REFERÊNCIAS}

1. ALVES GG, AERTS D. As práticas educativas em saúde e a Estratégia Saúde da Família. Ciência \& Saúde Coletiva, 2011;16:1.

2. BRASIL. Manual do Ministério da saúde. 2019. Disponível em: https://atencaobasica.saude.rs.gov.br/upload/arquivos/202001/03091259-nt-gestante-planificasus.pdf. Acessado em:2 de fevereiro de 2022.

3. BRASIL. Manual do Ministério da Saúde. 2014. Disponível em: https://pesquisa.bvsalud.org/bvsms/resource/pt/mis36844. Acessado em:27 de setembro de 2021.

4. BRASIL. Manual do Ministério da Saúde. 2013. Disponível em: $\begin{array}{llll}\text { https://bvsms.saude.gov.br/bvs/publicacoes/glossario_sgtes.pdf. Acessado em:27 de setembro de } 2021 . & \\ \text { BRASIL. Manual do Ministério da } & \text { Saúde. }\end{array}$

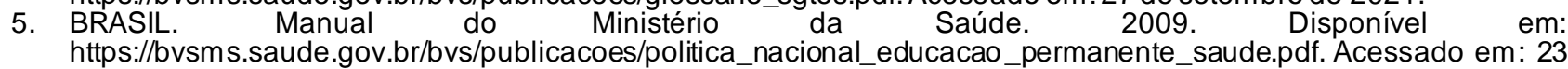
de janeiro de 2022.

6. BRASIL. Manual do Ministério da Saúde. 2007. Disponível em: https://bvsms.saude.gov.br/bvs/saudelegis/gm/2007/prt1996 2008 2007.html. Acessado em:05/02/2022.

7. CAMILO BS, et al. Grupo de gestantes: estratégias para o cuidado e educação em saúde. Biblioteca Lascasas, 2014;10:03.

8. CAVALCANTE JRC. Trabalho educativo em saúde no âmbito do Sistema Único de saúde (SUS). Dissertação (Mestrado em Educação) - Programa de Pós-Graduação em Educação. Universidade Federal de Goiás, Regional Jataí, 2019;140 p.

9. COUTINHO LRP, et al. Acolhimento na Atenção Primária à Saúde: revisão integrativa. Saúde Debate, 2015;39:514524.

10. DELFINO MRR, et al. O processo de cuidar participante com um grupo de gestantes: repercussões na saúde integral individual-coletiva. Ciência \& Saúde Coletiva, 2004;9:1057-1066.

11. DOMINGUES F, et al. Grupo de gestantes na Atenção Básica: espaço para construção do conhecimento e experiência na gestação. Revista da Faculdade de Ciências Médicas de Sorocaba, 2018; 20:150 -4.

12. HERBAS DTA. Improvisação: experiência com grupos de gestantes. Revista IGT na Rede, 2014; 11:3362-385.

13. HOGA LAK, REBERTE LM. Pesquisa-ação como estratégia para desenvolver grupo de gestantes: a percepção dos participantes. Revista Escola de Enfermagem USP, 2007;41:559-566.

14. MALUMBRES PC, BARRETO ICHC. Grupos de gestantes: o relato de uma experiência. Enfermagem Revista, 2016; 19:01.

15. NUNES GP, et al. Grupo de gestantes como ferramenta de instrumentalização e potencialização do cuidado. Cidadania em ação; Revista de Extensão e cultura, 2017; 1:1.

16. REBERTE LM, HOGA LAK. O desenvolvimento com um grupo de gestantes com a utilização da abordagem corporal. Texto e Contexto Enfermagem, 2005; 14:186-192.

17. SANTOS EAM, et al. Contribuições da educação permanente na atenção primária à saú de: uma revisão integrativa. Itinerarius Reflectionis, 2019;15:3.

18. SARTORI GS, VAN DER SAND ICP. Grupo de gestantes: espaço de conhecimentos, de trocas e de vínculos entre os participantes. Revista Eletrônica de Enfermagem, 2004;06:02.

19. VASCONCELOS EM. Redefinindo as práticas de saúde a partir da educação popular nos serviços de saúde. São Paulo: Hucitec, 2017;419 p.

20. VIEIRA NA et al. Trabalho interdisciplinar desenvolvido por profissionais de saúde em grupos de gestantes e/ou casais gravídicos (1996-2016). Revista Eletrônica [internet], 2019;10:51-53. 